\title{
Treatment of Oncological Patient in Terminal Stage with Two Different Hemodialysis - Case Report
}

Simona E Cioinac ${ }^{1 *}$ and T Lavalle ${ }^{2}$

${ }^{1}$ UO-Dialysis, CAL Bentivoglio, AUSL di Bologna, Italy

${ }^{2}$ Director UO Training, AUSL di Bologna, Italy

*Corresponding author: Simona E Cioinac, UO-Dialysis, CAL Bentivoglio, AUSL di Bologna, Italy, Tel: + 3494370660; E-mail: s.cioinac@ausI.bologna.it

Received date: June 13, 2016; Accepted date: July 11, 2016; Published date: July 12, 2016

Copyright: () 2016 Cioinac S, et al. This is an open-access article distributed under the terms of the Creative Commons Attribution License, which permits unrestricted use, distribution, and reproduction in any medium, provided the original author and source are credited

\begin{abstract}
Background: The number of patients with oncological diseases in hemodialysis is sharply increasing. These patients require hemodialysis and a personalized care that improves the tolerability, management of complications, pain, anxiety and fear of the patient and family. The literature presents approaches in different oncological diseases but there is still a dialysis treatment that allows greater tolerability. In this article, we describe the alternation of two different treatments and highlight the necessity of a personalized care process.
\end{abstract}

Case Presentation: Patient diagnosed with ovarian cancer, after countless series of complications, in the terminal phase requires service and haemodialysis customized. Prescribed treatment was: three treatments on-line hemodiafiltration of the duration 3:30 hours using monitors for intermittent haemodialysis. The fourth treatment catheter veno-venous hemodiafiltration of the duration 4 hours using monitors for continuous haemodialysis.

Conclusion: The case presented shows the need to identify a personalized care pathway for the management of cancer patients on haemodialysis and their families, good results. The alternation of the two treatments has allowed greater tolerance and fewer complications. This is just a clinical experience. Observational studies of RCTs (Random Trial Controls) could help clinics in understanding if the two alternate treatments can improve the quality of life, reduce pain and suffering.

Keywords: Personalized; Haemodialysis treatment; Oncological diseases.

\section{Introduction}

The last report of the Italian Registry of Dialysis and Transplant (RIDT 2010) revealed a prevalence of HD and PD of 788 patients per million population (PMP) and an incidence of 162/PMP (42,488 patients in haemodialysis, including 8,638 incident patients) [1,2]. The causes that lead to 'onset of a chronic renal disease in the terminal phase that requires haemodialysis are: cardiac diseases, diabetes, renal diseases, and oncological diseases [1,3]. Despite the many important scientific advances in oncology, which have certainly resulted in a marked improvement of the therapeutic approaches and increased survival of patients, cancer is still one of the most widespread diseases and a major cause of death in every part of the world. In the individual and collective imaginations, cancer is associated with severe physical and psychological suffering, death, diversity, guilt and shame. Cancer patients on haemodialysis have a very limited life expectancy and their suffering is greater than all other patients on haemodialysis and oncology $[4,5]$. In recent years, progress in cancer research and the development technologies led to longer life expectancies with clinical and political wide recognition of the 'importance of the quality of life of cancer patients. Regardless of the stage of the disease and the prognosis: clinical results may be affected by the state of mental and physical well, maintaining the quality of life including physical mechanisms of psychosocial and social is a priority medical and humanitarian [6-8]. In cancer patients on haemodialysis tolerability it is critical to ensure a decent quality of life $[8,9]$.

This report describes the case of a oncological patient in terminal stage in chronic renal failure haemodialysis treatment, the difficulties and his family together in the face of the neoplastic disease and the dialysis treatment and suggests the need to construct inside our dialysis centres a care pathway for oncological patient in haemodialysis treatment that allows a personalized assistance and a total care of the patient and family. It also describes the alternation of two different haemodialysis, HDF on-line (on-line hemodiafiltration) and CVVHDF (hemodiafiltration continuous veno-venous), which allow greater tolerability and the achievement of the dry weight of the patient and the object of anxiety and anguish.

\section{Case Presentation}

Mrs. SZ, age 63 years of Bosnian Serb origin, is transferred in our Dialysis Center of Support Limited Bentivoglio (Bentivoglio CAL) in December 2012 by the customer service Decentralized Maggiore (Maggiore CAD) where she began haemodialysis treatment (2010). On arrival the patient was stabilized and had to continue dialysis treatment at our centre. The history of the lady noted that in 2003 she was diagnosed with ovarian cancer with metastatic inguinal nodes, care was provided by ANT (National Cancer Association). Hysterectomy is performed in 2004 and in 2005 was subjected to three months of chemotherapy with CAELYX. A series of complications (2007 angioplasty+stent left renal artery, 2009 TVP axis axillo right subclavian based CVC) lead in 2010 to the beginning of haemodialysis 
using femoral venous catheter then left at the Centre CAD Maggiore. The treatment is bicarbonate haemodialysis (HD) biweekly, treatment time 3:30 hours. At the beginning of haemodialysis treatment, patient history does not reveal any pathology heart problem or diabetes. In 2010 is performed arteriovenous fistula venous haemodialysis left but in the same year the fistula does not work: at anatomical obstruction. In November 2010 it is packaged prosthetic fistula humerus cephalic left but in December 2010 she was diagnosed with complete thrombosis of the prosthesis. The patient continues treatment by CVC (Central Venous Catheter) right femoral. In March 2011, the pulmonary clinic visit and CT (Computed Tomography) highlighting calcified nodules compatible with metastasis. In April 2011 it is packaged new fistula between the proximal right brachial artery and venous branch deep malfunctioning. In August 2011, the lady is brought in Germany where it is packaged FAV cephalic humerus prosthetic left arm. In December 2011, occurs the thrombosis of the prosthesis and the patient returned to Germany where he is undergoes to the procedure of disobstruction. In March 2012, the abdominal CT highlights: vascular lesion oval solid roundish adherent to the posterior wall of the fast. In April 2012 thrombosis FAV prosthetic left: new revascularization procedure in Germany. In June 2012, the PET (positron emission tomography) highlights total body area of pathological accumulation in the abdominal lymph nodes compatible and always in June is diagnosed hypertensive heart disease. The triple revascularization performed in Germany in October 2012 not to good effect and in December of that year, in Germany, it is packed new fistula prosthetic left arm for haemodialysis. CAL arrived in Bentivoglio, in December 2012, the patient continues treatment with a twice-weekly HD treatment time of 3:30 hours until February 2013 when the rhythm becomes dialysis three times a week 3:30 hours of treatment. In the same period the body PET highlights accumulation radio medication at inter-aorta caval, and some level of mediastinal lymph nodes. The visits do not indicate cancer chemotherapy or other targeted therapies and continue the checks. In January 2014, the patient is visiting the Hospice Foundation Seragnoli Bentivoglio, begins morphine and continues to be followed by ANT home. From February 2014 to November 2014, the patient performs hemodialfiltration online (HDF online) for 3.30 hours three times a week and in November 2014 took the tetra weekly treatment, second treatment week is veno-venous continuous hemodiafiltration (CVVHDF) 4 hours.

\section{Discussion}

The traumatic event, cancer, invested abruptly Mrs. in 2003 by altering the equilibrium individual and interpersonal and evoking an atmosphere of uncertainty. It was a devastating existential test which involved all aspects of life: the relationship with her body, the meaning given to suffering, illness, death, as well as the family and social relationships. Not involved only her but her entire family. She, resident in Bosnia, comes in Italy, where her sons organized the start of treatment. The family has never given up in face of difficulties and after the first problems has activated in filled its own resources and the family has tried as far as possible to compensate for their limitations. The patient is brought several times in Germany, where his brother lives, to be subjected to interventions prosthetic packaging of FAV. One of the grandsons of Mrs. stops school and is dedicated to assistance day and night standing next to his grandmother during the haemodialysis sessions. The family does not accept, is not resigned to the progression of the neoplastic pathology claiming "when it is not evil, my mom is whit us and seems is good health". He asks the medical and nursing staff to put in place all the resources and knowledge to continue haemodialysis treatment. Being a Centre CAL, nurses are at the forefront in managing relations with the family, with the patient and the management of dialysis treatment. The nurses and doctors informed the family held on 'development of the disease and explain again that the hope in this case of advanced disease is a hope to the reality: maintain controlled the disease for as long as possible and with the least burden of suffering. The adaptation to the disease and the treatment depends largely on the quality of the relational 'team physician, who is the architect mainly by controlling the side effects of treatment, pain control, anxiety and depressive symptoms. This is possible through a personalized care of the patient, through information on the various aspects of the disease as well as through the evaluation of their needs, of his choice. This presupposes on the part of nursing staff also active listening of the history of the patient, his fantasies, his fears and defense mechanisms adopted to contain the anxiety. The experienced nurse dialysis is not only to manage a haemodialysis complex but must support the patient for 3:30-4 hours four times a week and the family. Support to the family during the terminal phase and critical to the nursing staff trained in dialysis but there is no care pathway in our Dialysis Centres of 'AUSL Bologna which provides a total care of the patient and family. The oncological disease causes a considerable intolerance during intermittent haemodialysis treatment. The management of dialysis treatment in a cancer patient is always difficult as tolerability is greatly reduced. In the literature are described several cases of oncological diseases in haemodialysis patients on haemodialysis or who develop neoplastic diseases. We are not aware of any other case where the treatment CVVHDF is used as the fourth weekly haemodialysis along with haemodialysis intermittent $\mathrm{HD}$ or online HDF lead to greater tolerance, indexes purification acceptable, longer survival than 6 months at home with your family. Earlier treatment HD biweekly 3:30 hours from positive effects on indices of purification here we are in possession and we can present them: KT/V=1.36 in 2012 when the patient arrived at our Centre. The vascular access shows a circulation of $8 \%$ calculated in December on the new fistula prosthetic left arm packed in Germany. The progressive course of neoplastic disease has involved the passage in HD treatment three times a week (April 2013) for duration of 3:30 in the treatment. The indices are maintained good purification $\mathrm{KT} / \mathrm{V}=1.23$ and the prosthesis highlights recirculation of $11.23 \%$. From the month of February 2014 until November 2014 (nine months of treatment) the patient performs on-line HDF, treatment time 3:30 hours, three times a week but the treatment does not give good results: $\mathrm{KT} / \mathrm{V}=0.96$, tolerability is low, supportable treatment time is $2: 30-3$ hours, agitation and anxiety take the upper hand throwing the patient and family in a in a situation of despair and suffering unmanageable. Manifest severe hypotensions that require stopping the treatment in emergency, severe pain that does not respond to painkiller, not reaching the dry weight. In November 2014 changes to the rhythm dialysis tetra weekly decay of general and poor tolerance to dialysis, problems with the dehydration of patient anuric. Relatives are constantly updated by the critical condition of the lady but ask to continue the treatments. The tetra weekly haemodialysis is composed of: $3 \mathrm{HDF}$ treatments online for a period of 3:30 and treatment veno-venous continuous hemodiafiltration (CVVHDF) of 4 hours. The treatment prescribed by your physician nephrologist centre is: CVVHDF online lasting 4 hours with the following indices: dialysate $2000 \mathrm{ml} / \mathrm{h}$, PBP $500 \mathrm{ml} / \mathrm{h}$, in post reinfusion $1000 \mathrm{ml} / \mathrm{h}$, weight loss, $500 \mathrm{~g} / \mathrm{h}, 180 \mathrm{QB} / 200$, Clexane 4000 beginning treatment. This type of treatment showed a greater tolerance, decreased pain 
Citation: Cioinac S, Lavalle T (2016) Treatment of Oncological Patient in Terminal Stage with Two Different Hemodialysis - Case Report. J Clin

Page 3 of 3

intradialytic, the achievement of the dry weight and improving the quality of life, $\mathrm{KT} / \mathrm{V}=1.03$.

\section{Conclusions}

The pathology associated with cancer in terminal phase haemodialysis does not allow the restoration; being a condition that is getting worse, it requires an arduous and continuous process of adaptation and personal assistance. Affected individuals can live several months or years in this particular condition of life; the disease, so it becomes a condition of life. Case presented demonstrates the effectiveness of the two alternate treatments, reduced pain and absence of personalized care and the absence appropriate training of expert nurses in hemodialysis. This is just a clinical experience. Our study sought to make a contribution in the management of these patients. The results bring out the need for further studies, perhaps observational or RCT, to see if the two types of treatment tolerability alternating increases and decreases complications. In conclusion we believe that care and haemodialysis personalized help cancer patients in the terminal phase of their lives by reducing the distress, anxiety and pain by giving time to the patient and family to face the inevitable.

\section{References}

1. De Santo NG, De Santo RM, Perna AF (2008) Surviving is not enough. Acts I International conference "Surviving is not enough: QOL in chronic kidney disease", Naples, 9-22.
2. (2010) Italian Registry of Dialysis and Transplant (RIDT), Italy.

3. Parsons JA, Davis AM (2004) Rehabilitation and quality-of-life issues in patients with extremity soft tissue sarcoma. Curr Treat Options Oncol 5: 477-488.

4. Mosconi P, Apolone G, Mingardi G (2008) Quality of life assessment and instruments in end- stage renal disease. J Nephrol 21: S107-S111.Jing L, Wu-Jun X, Feng T (2014) Palliative care for patients with malignancy and end-stage renal failure on peritoneal dialysis. Indian J Palliat Care 20: 137-141.

5. Upadhyay C, Cameron K, Murphy L, Battistella M (2014) Measuring pain in patients undergoing hemodialysis: a review of pain assessment tools. Clin Kidney J 7: 367-372.

6. Nagar VR, Birthi P (2015) Chronic opioid pain management for chronic kidney disease. J Pain Palliat Care Pharmacother 29: 48-50.

7. Douglas CA (2014) Palliative care for patients with advance chronic kidney disease. J R Coll Physicians Edinb 44: 224-231.

8. (2003) Distress management. Clinical practice guidelines. National Comprehensive Cancer Network. J Natl Compr Canc Netw 1: 344-374.

9. Meinero S, Tesio E, Bainotti S, Ferrando C, Gigliola G, et al. (2011) Evaluation of the quality of life of dialysis cunese. Nefrol 28: 72-79. 